\title{
DEALING WITH A TERRORIST ATTACK: A UNIVERSITY HOSPITAL PERSPECTIVE
}

\author{
Muhammad Shahid, Rifat Rehmani and M. Saleem Khan
}

Terrorist attacks must be dealt as an epidemic globally, as they usually cause multiple injured patients. Pakistan is also a target of such attacks. An explosives-packed car blew up near a bus taking Chinese engineers to work at Gwadar seaport at 8 AM on May 3, 2004. China is a key trading partner and ally of Pakistan and is funding $80 \%$ of the port project. This was the first time that Chinese workers have been targeted in Pakistan. Three Chinese were dead while 11 others were seriously injured. The injured were taken to the Gwadar Civil Hospital and later shifted to Aga Khan University Hospital (AKUH) Karachi at 2 PM.

In mass casualty events, the emergency medical services protocols that call for transport of patients in equitable distribution to appropriate centers is easily foiled because of confusion, route obstacles, and difficulty in communication. This was quite evident in New York City on $9 / 11^{1}$. The point at which the Emergency Department (ED) becomes overwhelmed often varies according to the time of day, the nature of injuries, and the amount of preparation time prior to the arrival of victims ${ }^{2}$. The Medical and Health Incident Management (MaHIM) System was recently developed as a model for planning a regional response that allows customizable organization of available assets for mass casualty response ${ }^{3}$. Joint
Commission on the Accreditation of Healthcare Organizations (JCAHO) requires that member hospitals have a written plan for the timely care of causalities arising from both external and internal disasters, and the hospital must document the rehearsal of these plans ${ }^{4}$. AKUH disaster plan was developed to mitigate confusion and chaos that can paralyze hospital's response during a disaster and includes common language, defined and predictable chain of management, flexible response, prioritized response, accountability of position function and documentation guidelines for accountability, cost recovery and is activated when multiple patients are received in the $E D^{5}$.

We present an epidemiologic description of physical injuries of the patients who survived the terrorist attack in the context of limited medical resources, and brought to the ED of a hospital.

Management of individual patients was reviewed from a pre-printed trauma form. Information on the nature of injuries, operative management and hospital course was recorded and data analyzed using the trauma registry. Trauma teams consisted of faculty and senior residents from Emergency Medicine, General Surgery, Neurosurgery, Orthopaedics and Anaesthesia arrived on time, and the support services performed well. They were divided into four teams consisting of one

TABLE I: NATURE OF INJURIES IN TERRORIST ATTACK PATIENTS

\begin{tabular}{|c|c|c|c|c|c|c|}
\hline Age (Years) & Laceration & Hematoma & Head injury & Ear perforation & Fracture & $\begin{array}{c}\text { Airway } \\
\text { obstruction }\end{array}$ \\
\hline 31 & $\sqrt{ }$ & & & & & \\
\hline 30 & $\sqrt{ }$ & $\sqrt{ }$ & $\sqrt{ }$ & & & \\
\hline 32 & $\sqrt{ }$ & & $\sqrt{ }$ & $\sqrt{ }$ & & \\
\hline 60 & & & & $\sqrt{ }$ & & \\
\hline 28 & $\sqrt{ }$ & & & $\sqrt{ }$ & & \\
\hline 33 & $\sqrt{ }$ & & & & & \\
\hline 35 & $\sqrt{ }$ & & & $\sqrt{ }$ & & \\
\hline 30 & $\sqrt{ }$ & & & & $\sqrt{ }$ & \\
\hline 36 & $\sqrt{ }$ & & & & $\sqrt{ }$ & \\
\hline 29 & $\sqrt{ }$ & & & & $\sqrt{ }$ & $\sqrt{ }$ \\
\hline 32 & $\sqrt{ }$ & & & & $\sqrt{ }$ & \\
\hline
\end{tabular}


member from each specialty.

All patients were male and their median age was 32 years. Two patients were unstable; one was intubated in ED and rushed to operating room after initial resuscitation where one patient had tracheostomy, facial laceration repaired, wound debridement of left arm and open reduction and internal fixation of left ulna was done, while the other patient had tracheostomy, exploratory laprotomy with splenectomy and open reduction and internal fixation of left radius and ulna. Nine patients had other injuries (lacerations and fractures). The nature of injuries is given in Table I. The mean length of stay in ED was 135 minutes.

All the 11 patients transferred to AKUH survived and discharged from the hospital after giving proper treatment. Disaster plan was tested in real-time and worked well on a holiday during rush hours. The relatively small sample size was a limitation in our study. The efficiency and feasibility of this disaster plan to handle a larger number of patients in a similar situation is yet not established, especially in public sector hospitals.

\section{REFERENCES}

1. Centers for Disease Control. Rapid assessment of injuries among survivors of the terrorist attack on the World Trade Centre - New York City, September 2001. MMWR 2002; 51:1.

2. Hogan DE, Waeckerle JF, Dire DJ, et al. Emergency department impact of the Oklahoma City terrorist bombing. Ann Emerg Med 1999; 34: 160-7.

3. Barbara JA, Macintyre AG. Medical and Health Incident Management (MaHIM) system. A comprehensive functional system description of Mass Casualty Medical and Health Incident Management. Institute for Crisis, Disaster, and Risk Management, The George Washington University. Washington D.C., October 2002.

4. Joint Commission on the Accreditation of Healthcare Organizations (JCAHO): Emergency Management Standard. Oak Brook Terrace, IL, JCAHO, 2001.

5. Aga Khan University Hospital Disaster Plan. Available at http://intranet/ss/PDFs/Emergency preparedness_Awareness.pdf. Accessed August 22, 2005.

AUTHOR AFFILIATION:
Dr. Muhammad Shahid (Corresponding Author)
Senior Instructor, Section of Emergency Medicine
Department of Medicine
Aga Khan University Hospital,
Stadium Road, Karachi - Pakistan.
Dr. Rifat Rehmani
Assistant Professor, Section of Emergency Medicine
Department of Medicine
Aga Khan University Hospital, Karachi.
M. Saleem Khan
Research Fellow, Department of Community Health Sciences
Aga Khan University, Karachi.

AUTHOR AFFILIATION:

Dr. Muhammad Shahid (Corresponding Author)

Senior Instructor, Section of Emergency Medicine

Department of Medicine

Aga Khan University Hospital,

Stadium Road, Karachi - Pakistan.

Dr. Rifat Rehmani

Assistant Professor, Section of Emergency Medicine

Department of Medicine

M. Saleem Khan

Research Fellow, Department of
Aga Khan University, Karachi. 